Research Article

\title{
Improved Lower Bound of LFMD with Applications of Prism-Related Networks
}

\author{
Muhammad Javaid ${ }^{D},{ }^{1}$ Hassan Zafar, ${ }^{1}$ Q. Zhu, ${ }^{2,3}$ and Abdulaziz Mohammed Alanazi ${ }^{4}{ }^{4}$ \\ ${ }^{1}$ Department of Mathematics, School of Science, University of Management and Technology, Lahore 54770, Pakistan \\ ${ }^{2}$ School of Mathematics and Staistics, Hunan Normal University, Changsha, Hunan 4100081, China \\ ${ }^{3}$ Department of Mathematics, School of Information Science and Engineering, Chengdu University, Chengdu 610106, China \\ ${ }^{4}$ Department of Mathematics, University of Tabuk, Tabuk, Saudi Arabia
}

Correspondence should be addressed to Muhammad Javaid; javaidmath@gmail.com

Received 22 March 2021; Revised 10 April 2021; Accepted 19 April 2021; Published 4 May 2021

Academic Editor: Sakander Hayat

Copyright $\odot 2021$ Muhammad Javaid et al. This is an open access article distributed under the Creative Commons Attribution License, which permits unrestricted use, distribution, and reproduction in any medium, provided the original work is properly cited.

\begin{abstract}
The different distance-based parameters are used to study the problems in various fields of computer science and chemistry such as pattern recognition, image processing, integer programming, navigation, drug discovery, and formation of different chemical compounds. In particular, distance among the nodes (vertices) of the networks plays a supreme role to study structural properties of networks such as connectivity, robustness, completeness, complexity, and clustering. Metric dimension is used to find the locations of machines with respect to minimum utilization of time, lesser number of the utilized nodes as places of the objects, and shortest distance among destinations. In this paper, lower bound of local fractional metric dimension for the connected networks is improved from unity and expressed in terms of ratio obtained by the cardinalities of the under-study network and the local resolving neighbourhood with maximum order for some edges of network. In the same context, the LFMDs of prism-related networks such as circular diagonal ladder, antiprism, triangular winged prism, and sun flower networks are computed with the help of obtained criteria. At the end, the bounded- and unboundedness of the obtained results is also shown numerically.
\end{abstract}

\section{Introduction}

For a connected network $G$, Salter introduced the concept of resolving (locating) set with the cardinality of minimum resolving set which is called the location number of $G$ [1]. Harary and Melter introduced the concept of metric dimension for the connected networks [2]. The concept of metric independence number $m i(G)$ of a graph $G$ is introduced by Currie and Oellermann [3]. The metric dimension has been applied to solve the problems involving percolation in hierarchical lattice [4], coin weighting, and robot navigation [5]. It is also applied in subject of chemistry to find the structures of chemical compounds having similar characteristics in functional groups. These functional groups play a vital role in chemical and pharmaceutical industries to predict the various chemical properties of the molecular compounds that are used in the drug discovery [6]. Metric dimension of graph was formulated as integer programming problem by Charterand et al. [6]. Fehar et al. studied the metric dimension of Cayley digraphs [7]. For further studies of metric dimension of convex polytopes, Caylay and Toeplitz networks, see [8-11].

Currie and Oellermann defined the concept of fractional metric dimension (FMD) as an optimal solution of the linear relaxation of the integer programming problem (IPP) [3]. Later, Faher et al. presented the identical calculation of IPP with the help of FMD [7]. Arguman and Matthew introduced many different properties of FMD for connected networks with respect to their order [12]. FMDs of hierarchical product of graphs were computed by Feng and Wang [13]. Liu et al. [14] computed the FMD of generalized Jahangir graph. The concept of local fractional metric 
dimension (LFMD) is introduced by Aisyah et al. [15]. They also computed it for the connected networks which are obtained by the operation of the corona product. The results for the LFMDs of some cycle-related networks and rotationally symmetric and planar networks can be found in $[16,17]$. Javaid et al. (2020) computed the sharp bounds of LFMD of connected networks and illustrated the obtained results with the help of wheel-related networks. They also compared the bounded- and unboundedness of the obtained results [18].

In this paper, lower bound of LFMD for connected networks is improved from unity and expressed in terms of ratio obtained by the cardinalities of the under-study network and the local resolving neighbourhood with maximum order for some edges of network. In the outcome of the obtained result, the LFMDs of prism-related networks as exact values and sharp bounds are computed. The rest of the article is organised as follows: Section 2 consists the preliminaries, Section 3 consists of main results of LFMD of connected networks, Section 4 deals with the local resolving neighbourhoods of prism-related networks, Section 5 presents LFMD of prism-related networks and Section 6 consists of conclusion and comparison among the main results.

\section{Preliminaries}

Let $G=(V(G), E(G))$ be a network with $V(G)$ and $E(G)$ as set of vertices and edges, respectively. A walk is defined as a sequence of alternating vertices and edges. A walk in which the vertices are all distinct is a path between vertices $a$ and $b$ and a closed path is called a cycle. For any two vertices $a$ and $b$ of $G$, the distance $d(a, b)$ is the length of shortest path $a \sim b$ in $G$. A pair of vertices $a$ and $b$ in a network is a connected pair if there is a path between them and the network is a connected network. For a connected network $G$ and $e=a b \in E(G)$, a vertex $x \in V(G)$ distinguishes two vertices $a$ and $b$ if $d(x, a)=d(x, b)$ is known as symmetric vertex. Moreover, $x$ resolves the edge $e$ in $G$ if $d(x, a) \neq d(x, b)$. For $D=\left\{a_{1}, a_{2}, a_{3}, \ldots, a_{n}\right\} \subseteq V(G)$ and $x \in V(G)$, the $k$-tuple metric form of $D$ in terms of $x$ is given by $r(x \mid D)=d\left(x, a_{1}\right), d\left(x, a_{2}\right), d\left(x, d_{3}\right), \ldots, d\left(x, a_{n}\right)$. The set $D$ becomes resolving set having $n$ elements of graph $G$ if each pair of vertices in $G$ bears a distinct metric form with respect to $D$. The resolving set with least number of vertices is referred as metric basis for $G$ and cardinality of such resolving set is called metric dimension of $G$ defined by

$$
\operatorname{dim}(G)=\min \{|D|: D \text { is resolving set of } G\} .
$$

For an edge $a b \in E(G)$, the local resolving neighbourhood (LRN) is defined as $\operatorname{LR}(a b)=\{x \in V(G): d$ $(x, a) \neq d(x, b)\}$, where $x \in V(G)$. A function is called an upper local resolving function (ULRF) if $f: V(G) \longrightarrow[0,1]$ and $f(\operatorname{LR}(a b)) \geq 1$ for each $\operatorname{LR}(a b)$ of $G$, where $f(\operatorname{LR}(a b))=\sum_{x \in \operatorname{LR}(a b)} f(x)$. On the other hand, a function is called lower local resolving function (LLRF) if $\Psi: V(G) \longrightarrow[0,1]$ and $\Psi(\operatorname{LR}(a b)) \leq 1$ for each $\operatorname{LR}(a b)$ of G, where $\Psi(\operatorname{LR}(a b))=\sum_{x \in \operatorname{LR}(a b)} \Psi(x)$. Then, LFMD is defined as $\operatorname{dim}_{l f}(G)=\tau$, where $\tau$ is $\min \{|f|: f$ which is the upper local minimal resolving function of $G\}$ or $\max \{|g|: g$ is the lower local maximal resolving function of $G\}$.

For $1 \leq i \leq n$, now we present some prism-related networks. The circular diagonal ladder $\left(\mathrm{CDL}_{n}\right)$ is obtained from prism network $D_{n}$ of order $2 n$ and size $5 n$ by adding some double crossing edges $a_{i} b_{(i+1)}$ and $a_{(i+1)} b_{i}$, as shown in Figure 1. The antiprism network $\left(A_{n}\right)$ of order $2 n$ and size $4 n$ is obtained by prism network $\left(D_{n}\right)$ by adding some crossing edges $a_{i} b_{(i+1)}$, see Figure 2. The sun flower network $\left(\mathrm{SF}_{n}\right)$ of order $2 n$ and size $3 n$, we mean a network, is isomorphic to the network obtained from $A_{n}$ by deleting edges $b_{i} b_{(i+1)}$, see Figure 3 [19].

Theorem 1 (see [18]). Let $G=(V(G), E(G))$ be a connected network. Let $L R(e)$ be a local resolving neighbourhood for the edge e of $G$. If $|L R(e) \cap Z| \geq \alpha, \forall e \in E(G)$, then

$$
1 \leq \operatorname{dim}_{l f}(G) \leq \frac{|Z|}{\alpha},
$$

where $Z=\cup\{L R(e):|L R(e)|=\alpha\}, \quad \alpha=\min \{|L R(e)|: e$ $\in E(G)\}$, and $2 \leq \alpha \leq|V(G)|$.

Proposition 1 (see [18]). Let $G=(V(G), E(G))$ be a connected network. For each $e \in E(G)$, if $|L R(e) \cap Z| \geq 2$, then $\operatorname{dim}_{l f}=(|Z| / 2)$, where $Z=\cup\{L R(e):|L R(e)|=2\}$, and $L R(e)$ is a $L R N$ set of $e \in E(G)$.

\section{Main Results}

Main results of LFMD are as follows.

Proposition 2. Let $G=(V(G), E(G))$ be a connected network and $L R(e)$ be the local resolving neighbourhood set of the edge $e$ of $G$. For $\beta=\max \{|L R(e)|: e=E(G)\}$, if $Y=\cup\{L R(e):|L R(e)|=\beta\} \subseteq V(G)$, then $|L R(e) \cap Y| \leq \beta$ for each local resolving neighbourhood $L R(e)$ of $G$.

Theorem 2. Let $G=(V(G), E(G))$ be a connected network and $L R(e)$ be the local resolving neighbourhood set. Then,

$$
\frac{|V(G)|}{\beta} \leq \operatorname{dim}_{l f}(G)
$$

where $\beta=\max \{|L R(e)|: e \in E(G)\}$ and $2 \leq \beta \leq|V(G)|$.

Proof. Define $\Phi: V(G) \longrightarrow[0,1]$ as $\phi(v)=(1 / \beta)$ for $v \in V(G)$. By Proposition 2, for $e \in E(G)$, we have

$$
\phi(\operatorname{LR}(e))=\sum_{x \in \operatorname{LR}(e)} \phi(x)=\sum_{x \in \operatorname{LR}(e) \cap V(G)} \frac{1}{\beta}=|\operatorname{LR}(e) \cap V(G)| \frac{1}{\beta} \leq 1 .
$$

This shows that $\phi$ is a lower local resolving function (LLRF). To show that $\phi$ is maximal, suppose on contrary, there exists another LLRF $\Psi$ such that $\Psi(x) \geq \Phi(x)$, where $\Psi(x) \neq \Phi(x)$, for at least one $x \in V(G) . \forall x \in \operatorname{LR}(e)$ such that $|\operatorname{LR}(e)|=\beta$, we have 


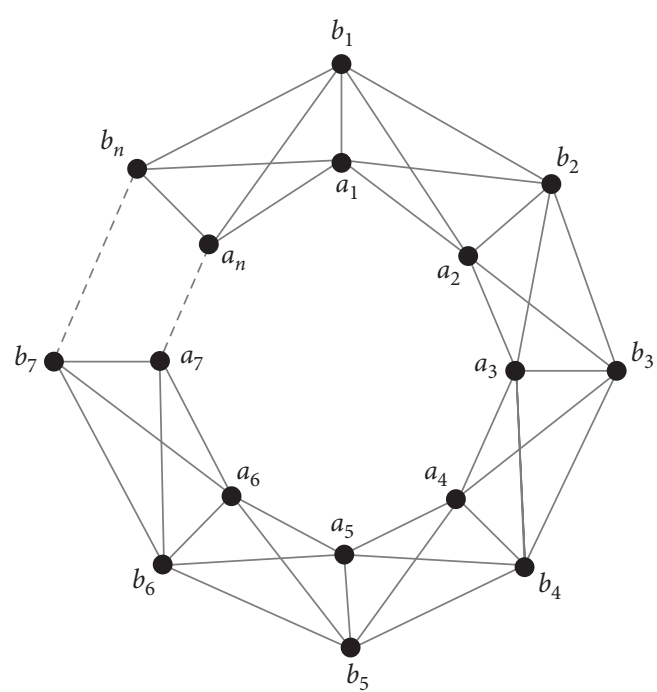

Figure 1: Circular diagonal ladder $\mathrm{CDL}_{n}$.

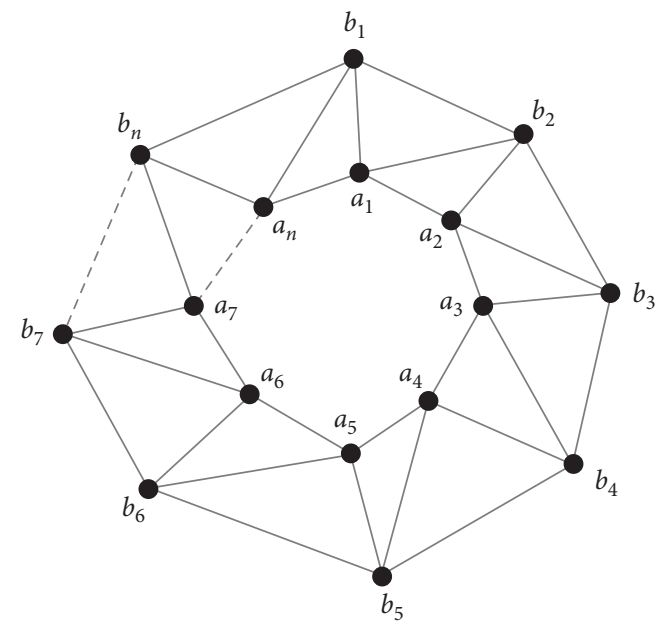

Figure 2: Antiprism $A_{n}$.

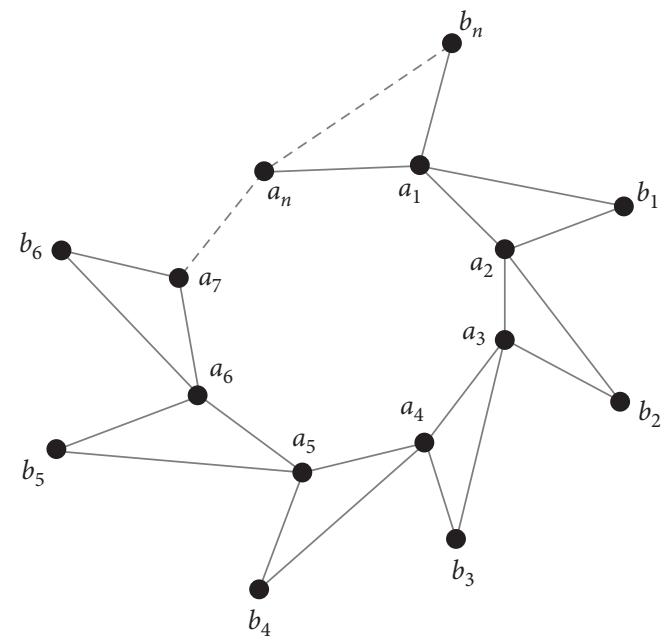

FIgURE 3: Sun flower network $\mathrm{SF}_{n}$.

$$
\Psi(\operatorname{LR}(e))=\sum_{x \in \operatorname{LR}(e)} \Psi(x)>\sum_{x \in \operatorname{LR}(e)} \phi(x)=1 .
$$

Thus, $\Psi(\operatorname{LR}(e))>1$. This shows that $\Psi$ is not LLRF and consequently $\phi$ is maximal LLRF. Let $\phi^{\prime}$ be another maximal LLRF of $G$. Then,

$$
\left|\phi^{\prime}\right|=\sum_{x \in V(G)} \phi^{\prime}(x) .
$$

Now, we consider three cases (i) $\phi^{\prime}(x)>(1 / \beta)$ for each $x \in V(G)$, (ii) $\phi^{\prime}(x) \leq(1 / \beta)$ for each $x \in V(G)$, and (iii) $\phi^{\prime}(x)>(1 / \beta)$ for some $x \in V(G)$.

Case 1: if $\phi^{\prime}(x)>(1 / \beta)$, for each $x \in V(G)$. For $\operatorname{LR}(e) \subseteq Y$ such that $|\operatorname{LR}(e)|=\beta$, we have $\phi^{\prime}(\operatorname{LR}(e)>1)$. This shows $\phi^{\prime}$ is not LLRF. Thus, this case does not hold.

Case 2: let $\phi^{\prime}(x) \leq(1 / \beta) \forall x \in V(G)$. Then,

$$
\left|\phi^{\prime}\right|=\sum_{x \in V(G)} \phi^{\prime}(x) \leq \frac{|V(G)|}{\beta}=|\phi| .
$$

Consequently,

$$
\operatorname{dim}_{l f}(G)=\frac{|V(G)|}{\beta} .
$$

Case 3: assume that $\phi^{\prime}(x)>(1 / \beta)$ for some $x \in V(G)$. Suppose that $S=\left\{t \in V(G): \phi^{\prime}(t)>(1 / \beta)\right\} \quad$ and $Y=\cup\{\operatorname{LR}(e):|\operatorname{LR}(e)|=\beta\}$. We note that $S \cap Y=\varnothing$; otherwise, for $|\operatorname{LR}(e)|=\beta, \phi^{\prime}(\operatorname{LR}(x))>1$ which implies that $\phi^{\prime}$ is not a LLRF. Consider

$$
\left|\phi^{\prime}\right|=\sum_{x \in V(G)} \phi^{\prime}(x)=\sum_{x \in Y} \phi^{\prime}(x)+\sum_{x \in V(G)-Y} \phi^{\prime}(x) .
$$

Since $\sum_{x \in V(G)-Y} \phi^{\prime}(x) \geq \sum_{x \in V(G)-Y} \phi(x)$, therefore,

$$
\begin{aligned}
\left|\phi^{\prime}\right|= & \sum_{x \in V(G)} \phi^{\prime}(x)=\sum_{x \in Y} \phi^{\prime}(x)+\sum_{x \in V(G)-Y} \phi^{\prime}(x) \geq \sum_{x \in Y} \phi(x) \\
& +\sum_{x \in V(G)-Y} \phi(x)=\frac{|V(G)|}{\beta}=|\phi| .
\end{aligned}
$$

Consequently,

$$
\operatorname{dim}_{l f}(G)=\left|\phi^{\prime}\right| \geq|\phi|=\frac{|V(G)|}{\beta} .
$$

Thus, from all the cases,

$\frac{|V(G)|}{\beta} \leq \operatorname{dim}_{l f}(G)$, which completes the proof.

Now, we present the following two corollaries as the direct consequences of the above result.

Corollary 1. Let $G=(V(G), E(G))$ be a connected network, $L R(e)$ be $L R N$ of $e \in E(G), \beta=\max \{|L R(e)|: e \in E(G)\}$, 
$\alpha=\min \{|L R(e)|: e \in E(G)\}$, and $X=\cup\{L R(e):|L R(e)|$ $=\alpha\}$. If $\alpha=\beta$ and $X=V(G)$, then

$$
\operatorname{dim}_{l f}(G)=\frac{|V(G)|}{\beta} .
$$

Proof. Since $\alpha=\beta$ and $X=V(G)$, therefore, by Theorem A, $\operatorname{dim}_{l f}(G)=(|V(G)| / \beta)$. Also, by Theorem 1, $\operatorname{dim}_{l f}$ $(G)=(|V(G)| / \beta)$. Consequently,

$$
\operatorname{dim}_{l f}(G)=\frac{|V(G)|}{\beta} .
$$

Corollary 2. Let $G=(V(G), E(G))$ be a connected network, $L R(e)$ be the $L R N$ of $e \in E(G), \beta=\max \{|L R(e)|: e \in E(G)\}$, $\alpha=\min \{|L R(e)|: e \in E(G)\}$, and $X=\cup\{L R(e):|L R(e)|=$ $\alpha\}$. If $\alpha=|X|$ and $\beta=|V(G)|$, then $\operatorname{dim}_{l f}(G)=1$.

Proof. Since $\beta=|V(G)|$, then by Theorem $2,1 \leq \operatorname{dim}_{l f}(G)$. Also, as $\alpha=|X|$, therefore, by Theorem $1, \operatorname{dim}_{l f}(G) \leq 1$. Consequently, $\operatorname{dim}_{l f}(G)=1$.

Remark 1. Corollary 2 strengthens the result proved in [18].

\section{LRNs of the Prism-Related Networks}

In this section, the local resolving neighbourhoods of prismrelated networks are classified.

Lemma 1. Let $C D L_{n}$ with $n \geq 4$ be a circular diagonal ladder network, where $n \cong 0(\bmod 2)$ and $\left|V\left(C D L_{n}\right)\right|=2 n$, where $1 \leq i \leq n$. We have

(a) $\left|L R\left(e_{i}\right)\right|=\left|L R\left(a_{i} b_{i}\right)\right|=2$ and $\left|\cup_{i=1}^{n} L R\left(e_{i}\right)\right|=2 n$,

(b) $\left|L R\left(e_{i}\right)\right|=\left|L R\left(b_{i} b_{(i+1)}\right)\right|$ and $\mid L R\left(b_{i} b_{(i+1)}\right) \cap \cup_{i=1}^{n} L R$ $\left(e_{i}\right)|\geq| L R\left(e_{i}\right) \mid$,

(c) $\left|L R\left(e_{i}\right)\right|=\left|L R\left(a_{i} a_{(i+1)}\right)\right|$ and $\mid L R\left(a_{i} a_{(i+1)}\right) \cap \cup_{i=1}^{n} L R$ $\left(e_{i}\right)|\geq| L R\left(e_{i}\right) \mid$,

(d) $\left|L R\left(e_{i}\right)\right|=\left|L R\left(a_{i} b_{(i+1)}\right)\right|$ and $\mid L R\left(a_{i} b_{(i+1)}\right) \cap \cup_{i=1}^{n} L R$ $\left(e_{i}\right)|\geq| L R\left(e_{i}\right) \mid$,

(e) $\left|L R\left(e_{i}\right)\right|=\left|L R\left(b_{i} a_{i+1}\right)\right|$ and $\mid L R\left(b_{i} a_{(i+1)}\right) \cap \cup_{i=1}^{n} L R$ $\left(e_{i}\right)|\geq| L R\left(e_{i}\right) \mid$,

(f) $\left|L R\left(e_{i}\right)\right|=\left|L R\left(a_{i} b_{i-1}\right)\right|$ and $\mid L R\left(a_{i} b_{(i-1)}\right) \cap \cup_{i=1}^{n} L R$ $\left(e_{i}\right)|\geq| L R\left(e_{i}\right) \mid$.

Proof. Assume that $a_{i}$ and $b_{i}$ are inner and outer vertices, respectively, for $1 \leq i \leq n$ and $n+1 \cong 1(\bmod n)$. We have the following:

(a) Consider $\operatorname{LR}\left(a_{i} b_{i}\right)=\left\{a_{i}, b_{i}\right\} \quad$ with $\left|\operatorname{LR}\left(e_{i}\right)\right|=\mid \operatorname{LR}$ $\left(a_{i} b_{i}\right) \mid=2$. Moreover, $\left|\cup_{i=1}^{n} \operatorname{LR}\left(e_{i}\right)\right|=\left|V\left(\mathrm{CDL}_{n}\right)\right|=$ $2 n$.

(b) $\operatorname{LR}\left(b_{i} b_{(i+1)}\right)=V\left(\mathrm{CDL}_{n}\right)-\left\{a_{i}, a_{(i+1)}\right\} \quad$ with $\quad$ LR $\left(b_{(i+1)} b_{i}\right)|=2 n-2>2=| \operatorname{LR}\left(e_{i}\right) \mid$ and $\mid \operatorname{LR}\left(b_{i} b_{(i+1)}\right)$ $\cap \cup_{i=1}^{n} \operatorname{LR}\left(e_{i}\right)|=2 n-2>2=| \operatorname{LR}\left(e_{i}\right) \mid$. (c) $\operatorname{LR}\left(a_{i} a_{(i+1)}\right)=V\left(\mathrm{CDL}_{n}\right)-\left\{b_{i}, b_{(i+1)}\right\} \quad$ with $\mid \operatorname{LR}\left(a_{i}\right.$ $\left.a_{(i+1)}\right)|=2 n-2>2=| \operatorname{LR}\left(e_{i}\right) \mid$ and $\mid \operatorname{LR}\left(a_{i} a_{(i+1)}\right) \cap$ $\cup_{i=1}^{n} \mathrm{LR}\left(e_{i}\right)|=2 n-2>2=| \operatorname{LR}\left(e_{i}\right) \mid$.

(d) $\operatorname{LR}\left(a_{i} b_{(i+1)}\right)=V\left(\mathrm{CDL}_{n}\right)-\left\{a_{(i+1)}, b_{i}\right\}$ with $\mid \operatorname{LR}\left(a_{i}\right.$ $\left.b_{(i+1)}\right)|=2 n-2>2=| \operatorname{LR}\left(e_{i}\right) \mid$ and $\mid \operatorname{LR}\left(a_{i} b_{(i+1)}\right) \cap$ $\cup_{i=1}^{n} \operatorname{LR}\left(e_{i}\right)|=2 n-2>2=| \operatorname{LR}\left(e_{i}\right) \mid$.

(e) $\operatorname{LR}\left(b_{i} a_{(i+1)}\right)=V\left(\mathrm{CDL}_{n}\right)-\left\{b_{(i+1)}, a_{i}\right\}$ with $\mid \operatorname{LR}\left(b_{i}\right.$ $\left.a_{(i+1)}\right)|=2 n-2>2=| \operatorname{LR}\left(e_{i}\right) \mid$ and $\mid \operatorname{LR}\left(b_{i} a_{(i+1)}\right) \cap$ $\cup_{i=1}^{n} \operatorname{LR}\left(e_{i}\right)|=2 n-2>2=| \operatorname{LR}\left(e_{i}\right) \mid$.

(f) $\operatorname{LR}\left(b_{i} a_{(i-1)}\right)=V\left(\mathrm{CDL}_{n}\right)-\left\{a_{(i-1)}, b_{(i+1)}\right\}$ with |LR $\left(b_{i} a_{(i+1)}\right)|=2 n-2>2=| \operatorname{LR}\left(e_{i}\right) \mid$ and $\mid \operatorname{LR}\left(b_{i} a_{(i-1)}\right)$ $\cap \cup_{i=1}^{n} \operatorname{LR}\left(e_{i}\right)|=2 n-2>2=| \operatorname{LR}\left(e_{i}\right) \mid$.

Lemma 2. Let $C D L_{n}$ with $n \geq 5$ be a circular diagonal ladder network, where $n \cong 1(\bmod 2)$ and $\left|V\left(C D L_{n}\right)\right|=2 n$, where $1 \leq i \leq n$. We have

(a) $\left|L R\left(e_{i}\right)\right|=\left|L R\left(a_{i} b_{i}\right)\right|=2$ and $\left|\cup_{i=1}^{n} L R\left(e_{i}\right)\right|=2 n$,

(b) $\left|L R\left(e_{i}\right)\right|<\left|L R\left(b_{i} b_{(i+1)}\right)\right|$ and $\mid L R\left(b_{i} b_{(i+1)}\right) \cap \cup_{i=1}^{n} L R$ $\left(e_{i}\right)|\geq| L R\left(e_{i}\right) \mid$,

(c) $\left|L R\left(e_{i}\right)\right|<\left|L R\left(a_{i} a_{(i+1)}\right)\right|$ and $\mid L R\left(a_{i} a_{(i+1)}\right) \cap \cup_{i=1}^{n} L R$ $\left(e_{i}\right)|\geq| L R\left(e_{i}\right) \mid$

(d) $\left|L R\left(e_{i}\right)\right|<\left|L R\left(a_{i} b_{(i+1)}\right)\right|$ and $\mid L R\left(a_{i} b_{(i+1)}\right) \cap \cup_{i=1}^{n} L R$ $\left(e_{i}\right)|\geq| L R\left(e_{i}\right) \mid$,

(e) $\left|L R\left(e_{i}\right)\right|<\left|L R\left(b_{i} a_{(i+1)}\right)\right|$ and $\mid L R\left(b_{i} a_{(i+1)}\right) \cap \cup_{i=1}^{n} L R$ $\left(e_{i}\right)|\geq| L R\left(e_{i}\right) \mid$,

(f) $\left|L R\left(e_{i}\right)\right|<\left|L R\left(a_{i} b_{i-1}\right)\right|$ and $\mid L R\left(a_{i} b_{(i-1)}\right) \cap \cup_{i=1}^{n} L R$ $\left(e_{i}\right)|\geq| L R\left(e_{i}\right) \mid$.

Proof. Assume that $a_{i}$ and $b_{i}$ are inner and outer vertices, respectively, for $1 \leq i \leq n$ and $n+1 \cong 1(\bmod n),(3 n+1 / 2) \cong$ $1(\bmod n)$. We have the following:

(a) Consider $\operatorname{LR}\left(a_{i} b_{i}\right)=\left\{a_{i}, b_{i}\right\} \quad$ with $\quad\left|\operatorname{LR}\left(e_{i}\right)\right|=$ $\left|\operatorname{LR}\left(a_{i} b_{i}\right)\right|=2 . \quad$ Moreover, $\quad\left|\cup_{i=1}^{n} \operatorname{LR}\left(e_{i}\right)\right|=\mid V($ $\left.\mathrm{CDL}_{n}\right) \mid=2 n$.

(b) As $\operatorname{LR}\left(b_{i} b_{(i+1)}\right)=V\left(\mathrm{CDL}_{n}\right)-\left\{b_{(n+2 i+1 / 2)}, a_{(n+2 i+1 / 2)}\right.$, $\left.a_{i}, a_{(i+1)}\right\}$ with $\left|\operatorname{LR}\left(b_{i} b_{(i+1)}\right)\right|=2 n-4>2=\left|\operatorname{LR}\left(e_{i}\right)\right|$ and $\left|\operatorname{LR}\left(b_{i} b_{(i+1)}\right) \cap \cup_{i=1}^{n} \operatorname{LR}\left(e_{i}\right)\right|=2 n-4>2=$ $\left|\operatorname{LR}\left(e_{i}\right)\right|$.

(c) As $\operatorname{LR}\left(a_{i} a_{(i+1)}\right)=V\left(\mathrm{CDL}_{n}\right)-\left\{a_{(n+2 i+1 / 2)}, b_{(n+2 i+1 / 2)}\right.$, $\left.b_{i}, b_{(i+1)}\right\}$ with $\left|\operatorname{LR}\left(a_{i} a_{(i+1)}\right)\right|=2 n-4>2=\left|\operatorname{LR}\left(e_{i}\right)\right|$ and $\left|\operatorname{LR}\left(a_{i} a_{(i+1)}\right) \cap \cup_{i=1}^{n} \operatorname{LR}\left(e_{i}\right)\right|=2 n-4>2=$ $\left|\mathrm{LR}\left(e_{i}\right)\right|$.

(d) As $\operatorname{LR}\left(a_{i} b_{(i+1)}\right)=V\left(\mathrm{CDL}_{n}\right)-\left\{a_{(n+2 i+1 / 2)}, b_{(n+2 i+1 / 2)}\right.$, $\left.a_{(i+1)}, b_{i}\right\}$ with $\left|\operatorname{LR}\left(a_{i} b_{(i+1)}\right)\right|=2 n-4>2=\left|\operatorname{LR}\left(e_{i}\right)\right|$ and $\left|\operatorname{LR}\left(a_{i} b_{(i+1)}\right) \cap \cup_{i=1}^{n} \operatorname{LR}\left(e_{i}\right)\right|=2 n-4>2=\mid \mathrm{LR}$ $\left(e_{i}\right) \mid$.

(e) As $\operatorname{LR}\left(b_{i} a_{(i+1)}\right)=V\left(\mathrm{CDL}_{n}\right)-\left\{a_{(i)}, b_{(i+1)}, a_{(n+2 i+1 / 2)}\right.$, $\left.b_{(n+2 i+1 / 2)}\right\}$ with $\left|\operatorname{LR}\left(b_{i} a_{(i+1)}\right)\right|=2 n-4>2=\left|\operatorname{LR}\left(e_{i}\right)\right|$ and $\left|\operatorname{LR}\left(b_{i} a_{(i+1)}\right) \cap \cup_{i=1}^{n} \operatorname{LR}\left(e_{i}\right)\right|=2 n-4>2=\mid \mathrm{LR}$ $\left(e_{i}\right) \mid$.

(f) As $\operatorname{LR}\left(a_{i} b_{(i-1)}\right)=V\left(\mathrm{CDL}_{n}\right)-\left\{a_{i-1}, b_{i-1}, a_{(n+2 i+1 / 2)}\right.$, $\left.b_{(n+2 i+1 / 2)}\right\}$ with $\left|\operatorname{LR}\left(b_{i} a_{(i+1)}\right)\right|=2 n-4>2=\left|\operatorname{LR}\left(e_{i}\right)\right|$ 
and $\left|\operatorname{LR}\left(b_{i} a_{(i+1)}\right) \cap \cup_{i=1}^{n} \operatorname{LR}\left(e_{i}\right)\right|=2 n-4>2=\mid \mathrm{LR}$ $\left(e_{i}\right)$.

Lemma 3. Let $A_{n}$ with $n \geq 4$ be an antiprism network, where $n \cong 0(\bmod 2)$ and $\left|V\left(A_{n}\right)\right|=2 n$ and $1 \leq i \leq n$. We have

(a) $\left|\operatorname{LR}\left(e_{i}\right)\right|=\left|\operatorname{LR}\left(a_{i} b_{i}\right)\right|$ and $\left|\operatorname{LR}\left(a_{i} b_{(i+1)}\right)\right|=n$ and $\left|\cup_{i=1}^{n} \operatorname{LR}\left(e_{i}\right)\right|=2 n$,

(b) $\left|\operatorname{LR}\left(e_{i}\right)\right|=\left|\operatorname{LR}\left(b_{i} b_{(i+1)}\right)\right| \quad$ and $\mid L R\left(b_{i} b_{(i+1)}\right) \cap \cup_{i=1}^{n}$ $\operatorname{LR}\left(e_{i}\right)|\geq| \operatorname{LR}\left(e_{i}\right) \mid$,

(c) $\left|L R\left(e_{i}\right)\right|=\left|\operatorname{LR}\left(a_{i} a_{(i+1)}\right)\right| \quad$ and $\quad \mid L R\left(a_{i} a_{(i+1)}\right) \cap \cup_{i=1}^{n}$ $\operatorname{LR}\left(e_{i}\right)|\geq| \operatorname{LR}\left(e_{i}\right) \mid$.

Proof. Assume that $a_{i}$ and $b_{i}$ are inner and outer vertices, respectively, for $1 \leq i \leq n$, where $n+1 \cong 1(\bmod 2)$, $(3 n+2 / 2) \cong 1(\bmod n)$, and $(3 n / 2) \cong 1(\bmod n)$. We have

(a) $\operatorname{LR}\left(e_{i}\right)=\operatorname{LR}\left(a_{i} b_{i}\right)=\left\{b_{i}, \quad b_{(n+2 i+2 / 2)}, b_{(n+2 i+2 / 2)+1}\right.$, $b_{(n+2 i+2 / 2)+2}, \ldots, b_{(n+i-1)}, a_{i}, a_{(i+1)}, a_{(i+2)}, \ldots, a_{(n+2 i-}$

$2 / 2)\}$ and $\operatorname{LR}\left(a_{i} b_{(i+1)}\right)=\left\{b_{(i+1)}, b_{(i+2)}, b_{(i+3)}, \ldots\right.$, $b_{(2 i+n / 2)}, a_{i}, a_{(n+2 i+2 / 2)}, a_{(n+2 i+2 / 2)+1}, a_{(n+2 i+2 / 2)+2}, \ldots$,

$\left.a_{(n+i-1)}\right\} \quad$ with $\quad\left|\operatorname{LR}\left(e_{i}\right)\right|=n$. Moreover, $\left|\cup_{i=1}^{n} \operatorname{LR}\left(e_{i}\right)\right|=\left|V\left(A_{n}\right)\right|=2 n$,

(b) As $\operatorname{LR}\left(b_{i} b_{(i+1)}\right)=V\left(A_{n}\right)-\left\{a_{i}, a_{(n+2 i / 2)}\right\}$ with $\mid \operatorname{LR}\left(b_{i}\right.$ $\left.b_{(i+1)}\right)|=2 n-2>n=| \operatorname{LR}\left(e_{i}\right) \mid$ and $\left.\mid \operatorname{LR}\left(b_{i} b_{(i+1}\right)\right) \cap$ $\cup_{i=1}^{n} \operatorname{LR}\left(e_{i}\right)|=2 n-2>n=| \operatorname{LR}\left(e_{i}\right) \mid$,

(c) As $\operatorname{LR}\left(a_{i} a_{(i+1)}\right)=V\left(A_{n}\right)-\left\{b_{(i+2)}, b_{(n+2 i+2 / 2)}\right\}$ with $\left|\operatorname{LR}\left(a_{i} a_{(i+1)}\right)\right|=2 n-2>n=\left|\operatorname{LR}\left(e_{i}\right)\right|$ and $\mid \operatorname{LR}\left(a_{i} a\right.$ $(i+1)) \cap \cup_{i=1}^{n} \operatorname{LR}\left(e_{i}\right)|=2 n-2>n=| \operatorname{LR}\left(e_{i}\right) \mid$.

Lemma 4. Let $A_{n}$ with $n \geq 3$ be an antiprism network, where $\left|V\left(A_{n}\right)\right|=2 n$ with $n \cong 1(\bmod 2)$. For $1 \leq i \leq n$, we have

(a) $\left|\operatorname{LR}\left(e_{i}\right)\right|=\left|\operatorname{LR}\left(a_{i} b_{i}\right)\right|$ and $\left|\operatorname{LR}\left(a_{i} b_{(i+1)}\right)\right|=n+1$ and $\left|\cup_{i=1}^{n} L R\left(e_{i}\right)\right|=2 n$,

(b) $\left|L R\left(e_{i}\right)\right|=\left|L R\left(b_{i} b_{(i+1)}\right)\right|$ and $\mid L R\left(b_{i} b_{(i+1)}\right) \cap \cup_{i=1}^{n} L R$ $\left(e_{i}\right)|\geq| \operatorname{LR}\left(e_{i}\right) \mid$,

(c) $\left|L R\left(e_{i}\right)\right|=\left|L R\left(a_{i} a_{(i+1)}\right)\right|$ and $\mid L R\left(a_{i} a_{(i+1)}\right) \cap \cup_{i=1}^{n} L R$ $\left(e_{i}\right)|\geq| \operatorname{LR}\left(e_{i}\right) \mid$.

Proof. Assume that $a_{i}$ and $b_{i}$ are inner and outer vertices, respectively, for $1 \leq i \leq n$, where $n+1 \cong 1(\bmod 2)$, $(3 n+1 / 2) \cong 1(\bmod n), \quad(3 n-1 / 2) \cong 1(\bmod n), \quad$ and $2 n-1 \cong 1(\bmod 2)$. We have

(a) $\operatorname{LR}\left(a_{i} b_{i}\right)=\left\{a_{i}, a_{(i+1)}, a_{(i+2)}, \ldots, a_{(n+2 i-1 / 2)}, b_{i}, \quad b_{(n+2 i}\right.$ $\left.+1 / 2), b_{(n+2 i+3 / 2)}, \ldots, b_{(n+i-1)}\right\} \quad$ and $\operatorname{LR}\left(a_{i} b_{(i+1)}\right)=$ $\left\{b_{(i+1)}, b_{(i+2)}, \ldots, b_{(n+2 i+1 / 2)}, a_{i}, a_{(n+2 i+1 / 2)}, a_{(n+2 i+1 / 2)}\right.$, $\left.\ldots, a_{(n+i-1)}\right\}$ with $\left|\operatorname{LR}\left(e_{i}\right)\right|=n+1$. Moreover, $\left|\cup_{i=1}^{n} \operatorname{LR}\left(e_{i}\right)\right|=\left|V\left(A_{n}\right)\right|=2 n$. (b) $\operatorname{LR}\left(a_{i} a_{(i+1)}\right)=V\left(A_{n}\right)-\left\{\left(a_{(n+2 i+1 / 2)}, b_{(i+1)}\right)\right\}$ and |LR $\left(a_{i} a_{(i+1)}\right)|=2 n-2>n+1=| \operatorname{LR}\left(e_{i}\right) \mid$.

(c) $\operatorname{LR}\left(b_{i} b_{(i+1)}\right)=V\left(A_{n}\right)-\left\{a_{i}, b_{(n+2 i+1 / 2)}\right\}$ and $\mid \operatorname{LR}\left(b_{i}\right.$ $\left.b_{(i+1)}\right)|=2 n-2>n+1=| \operatorname{LR}\left(e_{i}\right) \mid$.

Lemma 5. Let $S F_{n}$ with $n \geq 3$ be a sun flower network, where $\left|V\left(S F_{n}\right)\right|=2 n$ and $n \cong 1(\bmod 2)$. For $1 \leq i \leq n$, we have

(a) $\left|L R\left(e_{i}\right)\right|=\left|\operatorname{LR}\left(a_{i} b_{i}\right)\right|$ and $\left|L R\left(b_{i} a_{(i+1)}\right)\right|=n+1$ and $\left|\cup_{i=1}^{n} L R\left(e_{i}\right)\right|=2 n$,

(b) $\left|L R\left(e_{i}\right)\right|=\left|\operatorname{LR}\left(a_{i} a_{i+1}\right)\right|$ and $\mid L R\left(a_{i} a_{(i+1)}\right) \cap \cup_{i=1}^{n} L R$ $\left(e_{i}\right)|\geq| \operatorname{LR}\left(e_{i}\right) \mid$.

Proof. Assume that $a_{i}$ and $b_{i}$ are inner and outer vertices, respectively, for $1 \leq i \leq n$, where $n+1 \cong 1(\bmod n)$, $n+2 \cong 1(\bmod n)$,

$(3 n+1 / 2) \cong 1(\bmod n)$, $(3 n-1 / 2) \cong 1(\bmod n)$, and $2 n-1 \cong 1(\bmod n)$. Now, we have

(a) $\operatorname{LR}\left(a_{i} b_{i}\right)=\left\{a_{i}, a_{(n+} \quad 2 i+1 / 2\right), a_{(n+2 i+1 / 2)+1}, \ldots, a$ $\left.\left.{ }_{(n+i-1)}, b_{i}, b_{(n+2 i+} 1 / 2\right), b_{(n+2 i+1 / 2)+1}, \ldots, b_{(n+i-1)}\right\}$ and $\operatorname{LR}\left(b_{i} a_{(i+1)}\right)=\left\{b_{i}, b_{(i+1)}, b_{(i+2)}, \ldots, b_{((n+2 i+1 / 2))}, a_{(i+1)}\right.$, $\left.a_{(i+2)}, a_{(i+3)}, \ldots, a_{(n+i-1)}\right\} \quad$ with $\quad\left|\operatorname{LR}\left(e_{i}\right)\right|=n+1$. Moreover, $\left|\cup_{i=1}^{n} \operatorname{LR}\left(e_{i}\right)\right|=\left|V\left(\mathrm{SF}_{n}\right)\right|=2 n$.

(b) $\operatorname{LR}\left(a_{i} a_{(i+1)}\right)=V\left(\mathrm{SF}_{n}\right)-\left\{a_{(n+2 i+1 / 2)}, b_{i}\right\}$ and |LR $\left(a_{i} a_{(i+1)}\right) \mid=2 n-2$ with $\left|\operatorname{LR}\left(a_{i} a_{(i+1)}\right)\right|=2 n-2>n+$ $1=\left|\operatorname{LR}\left(e_{i}\right)\right|$ and $\left|\operatorname{LR}\left(a_{i} a_{(i+1)}\right) \cap \cup_{i=1}^{n} \operatorname{LR}\left(e_{i}\right)\right|=2 n-2$ $>n+1=\left|\operatorname{LR}\left(e_{i}\right)\right|$.

Lemma 6. Let $S F_{n}$ with $n \geq 4$ be a sun flower network, where $\left|V\left(S F_{n}\right)\right|=2 n$ and $n \cong 0(\bmod 2)$. For $1 \leq i \leq n$, we have

(a) $\left|L R\left(e_{i}\right)\right|=\left|L R\left(a_{i} b_{i}\right)\right|$ and $\left|L R\left(b_{i} a_{(i+1)}\right)\right|=n+1$ and $\left|\cup_{i=1}^{n} L R\left(e_{i}\right)\right|=2 n$,

(b) $\left|L R\left(e_{i}\right)\right|=\left|\operatorname{LR}\left(a_{i} a_{(i+1)}\right)\right|$ and $\mid L R\left(a_{i} a_{(i+1)}\right) \cap \cup_{i=1}^{n} L R$ $\left(e_{i}\right)|\geq| \operatorname{LR}\left(e_{i}\right) \mid$.

Proof. Assume that $a_{i}$ and $b_{i}$ are inner and outer vertices, respectively, for $1 \leq i \leq n$, where $n+1 \cong 1(\bmod n)$, $n+2 \cong 1(\bmod n), \quad(3 n+1 / 2) \cong 1(\bmod n), \quad(3 n / 2) \cong 1(\bmod$ $n)$, and $2 n-1 \cong 1(\bmod n)$. We have

(a) $2 n-1 \cong 1(\bmod n)$ and $\operatorname{LR}\left(b_{i} a_{(i+1)}\right)=\left\{b_{i}, b_{(i+1)}\right.$, $\left.b_{(i+2)}, \ldots, b_{(n+2 i / 2)}, a_{(i+1)}, \quad a_{(i+2)}, a_{(i+3)}, \ldots, a_{(n+2 i / 2)}\right\}$ with $\left|\operatorname{LR}\left(e_{i}\right)\right|=n+1$. Moreover, $\mid \cup_{i=1}^{n}$ $\operatorname{LR}\left(e_{i}\right)|=| V\left(\mathrm{SF}_{n}\right) \mid=2 n$.

(b) $\operatorname{LR}\left(a_{i} a_{(i+1)}\right)=V\left(\mathrm{SF}_{n}\right)-\left\{b_{(n+2 i / 2)}, b_{i}\right\} \quad$ and $\quad$ LR $\left(a_{i} a_{i+1}\right) \mid=2 n-2$ with $\left|\operatorname{LR}\left(a_{i} a_{(i+1)}\right)\right|=2 n-2>n+1$ $=\left|\operatorname{LR}\left(e_{i}\right)\right|$ and $\left|\operatorname{LR}\left(a_{i} a_{i+1}\right) \cap \cup_{i=1}^{n} \operatorname{LR}\left(e_{i}\right)\right|=2 n-2>$ $n+1=\left|\operatorname{LR}\left(e_{i}\right)\right|$. 


\section{LFMD of Prism-Related Networks}

The LFMD of prism-related networks is computed as follows.

Theorem 3. Let $C D L_{n}$ with $n \geq 4$ be a circular diagonal ladder network and $\left|V\left(C D L_{n}\right)\right|=2 n$. Then, $\operatorname{dim}_{l f}\left(C D L_{n}\right)=n$.

Proof. Case 1: For $n=4$, LRNs are as follows:

$$
\begin{aligned}
& \mathrm{LR}_{1}=\mathrm{LR}\left(b_{1} b_{2}\right)=V\left(\mathrm{CDL}_{4}\right)-\left\{a_{1}, a_{2}\right\}, \\
& \mathrm{LR}_{2}=\mathrm{LR}\left(b_{2} b_{3}\right)=V\left(\mathrm{CDL}_{4}\right)-\left\{a_{2}, a_{3}\right\} \text {, } \\
& \mathrm{LR}_{3}=\mathrm{LR}\left(b_{3} b_{4}\right)=V\left(\mathrm{CDL}_{4}\right)-\left\{a_{3}, a_{4}\right\}, \\
& \mathrm{LR}_{1}=\operatorname{LR}\left(b_{1} b_{2}\right)=V\left(\mathrm{CDL}_{4}\right)-\left\{a_{1}, a_{2}\right\} \text {, } \\
& \mathrm{LR}_{5}=\operatorname{LR}\left(a_{1} a_{2}\right)=V\left(\mathrm{CDL}_{4}\right)-\left\{b_{1}, b_{2}\right\} \text {, } \\
& \mathrm{LR}_{6}=\operatorname{LR}\left(a_{2} a_{3}\right)=V\left(\mathrm{CDL}_{4}\right)-\left\{b_{2}, b_{3}\right\} \text {, } \\
& \mathrm{LR}_{7}=\operatorname{LR}\left(a_{3} a_{4}\right)=V\left(\mathrm{CDL}_{4}\right)-\left\{b_{3}, b_{4}\right\}, \\
& \mathrm{LR}_{8}=\operatorname{LR}\left(a_{4} a_{1}\right)=V\left(\mathrm{CDL}_{4}\right)-\left\{b_{4}, b_{1}\right\} \text {, } \\
& \mathrm{LR}_{9}=\operatorname{LR}\left(a_{1} b_{2}\right)=V\left(\mathrm{CDL}_{4}\right)-\left\{a_{2}, b_{1}\right\} \text {, } \\
& \mathrm{LR}_{10}=\operatorname{LR}\left(a_{2} b_{3}\right)=V\left(\mathrm{CDL}_{4}\right)-\left\{a_{3}, b_{2}\right\} \text {, } \\
& \mathrm{LR}_{11}=\operatorname{LR}\left(a_{3} b_{4}\right)=V\left(\mathrm{CDL}_{4}\right)-\left\{a_{4}, b_{3}\right\} \text {, } \\
& \mathrm{LR}_{12}=\operatorname{LR}\left(a_{4} b_{1}\right)=V\left(\mathrm{CDL}_{4}\right)-\left\{a_{1}, b_{4}\right\} \text {, } \\
& \mathrm{LR}_{13}=\operatorname{LR}\left(a_{2} b_{1}\right)=V\left(\mathrm{CDL}_{4}\right)-\left\{a_{1}, b_{2}\right\} \text {, } \\
& \mathrm{LR}_{14}=\operatorname{LR}\left(a_{3} b_{2}\right)=V\left(\mathrm{CDL}_{4}\right)-\left\{a_{2}, b_{3}\right\} \text {, } \\
& \mathrm{LR}_{15}=\operatorname{LR}\left(a_{4} b_{3}\right)=V\left(\mathrm{CDL}_{4}\right)-\left\{a_{3}, b_{4}\right\} \text {, } \\
& \mathrm{LR}_{16}=\operatorname{LR}\left(a_{1} b_{4}\right)=V\left(\mathrm{CDL}_{4}\right)-\left\{a_{4}, b_{1}\right\} \text {, } \\
& \mathrm{LR}_{17}=\operatorname{LR}\left(b_{1} a_{2}\right)=V\left(\mathrm{CDL}_{4}\right)-\left\{b_{2}, a_{1}\right\} \text {, } \\
& \mathrm{LR}_{18}=\mathrm{LR}\left(b_{2} a_{3}\right)=V\left(\mathrm{CDL}_{4}\right)-\left\{b_{3}, a_{2}\right\} \text {, } \\
& \mathrm{LR}_{19}=\mathrm{LR}\left(b_{3} a_{4}\right)=V\left(\mathrm{CDL}_{4}\right)-\left\{b_{4}, a_{3}\right\} \text {, } \\
& \mathrm{LR}_{20}=\mathrm{LR}\left(b_{4} a_{1}\right)=V\left(\mathrm{CDL}_{4}\right)-\left\{b_{1}, a_{4}\right\} \text {, } \\
& \operatorname{LR}_{21}=\operatorname{LR}\left(a_{1} b_{1}\right)=\left\{a_{1}, b_{1}\right\}, \\
& \mathrm{LR}_{22}=\operatorname{LR}\left(a_{2} b_{2}\right)=\left\{a_{2}, b_{2}\right\}, \\
& \mathrm{LR}_{23}=\operatorname{LR}\left(a_{3} b_{3}\right)=\left\{a_{3}, b_{3}\right\}, \\
& \operatorname{LR}_{24}=\operatorname{LR}\left(a_{4} b_{4}\right)=\left\{a_{4}, b_{4}\right\} \text {. }
\end{aligned}
$$

As, for $1 \leq i \leq 4,\left|\operatorname{LR}\left(e_{i}\right)\right|$ is 2 such that $\left|\operatorname{LR}\left(e_{i}\right)\right|<\left|\operatorname{LR}\left(e_{s}\right)\right|$, where $1 \leq s \leq 20$. Moreover, $\cup_{i=1}^{4} L R\left(e_{i}\right)=8$ and $\left|\mathrm{LR}_{s} \cap \cup_{i=1}^{4} \mathrm{LR}_{i}\right|=\left|\mathrm{LR}_{i}\right|=2$. Define $\phi: V\left(\mathrm{CDL}_{4}\right) \longrightarrow$ $[0,1]$ as $\phi(x)=(1 / 2)$ is a LRF with minimum cardinality for each $x \in V\left(\mathrm{CDL}_{4}\right)$. Consequently, by Preposition 1 , $\operatorname{dim}_{l f}\left(\mathrm{CDL}_{4}\right)=\left(\left|V\left(\mathrm{CDL}_{4}\right)\right| / 2\right)=4$.

Case 2: For $n=5$, LRNs are as follows:

$$
\begin{aligned}
& \mathrm{LR}_{1}=\operatorname{LR}\left(b_{1} b_{2}\right)=V\left(\mathrm{CDL}_{5}\right)-\left\{b_{4}, a_{4}, a_{1}, a_{2}\right\}, \\
& \mathrm{LR}_{2}=\operatorname{LR}\left(b_{2} b_{3}\right)=V\left(\mathrm{CDL}_{5}\right)-\left\{b_{5}, a_{5}, a_{2}, a_{3}\right\}, \\
& \mathrm{LR}_{3}=\operatorname{LR}\left(b_{3} b_{4}\right)=V\left(\mathrm{CDL}_{5}\right)-\left\{b_{6}, a_{6}, a_{3}, a_{4}\right\}, \\
& \mathrm{LR}_{4}=\operatorname{LR}\left(b_{4} b_{5}\right)=V\left(\mathrm{CDL}_{5}\right)-\left\{b_{2}, a_{2}, a_{4}, a_{5}\right\}, \\
& \mathrm{LR}_{5}=\operatorname{LR}\left(b_{5} b_{1}\right)=V\left(\mathrm{CDL}_{5}\right)-\left\{b_{3}, a_{1}, a_{3}, a_{5}\right\}, \\
& \mathrm{LR}_{6}=\operatorname{LR}\left(a_{1} a_{2}\right)=V\left(\mathrm{CDL}_{5}\right)-\left\{b_{4}, a_{4}, b_{1}, b_{2}\right\}, \\
& \mathrm{LR}_{7}=\operatorname{LR}\left(a_{2} a_{3}\right)=V\left(\mathrm{CDL}_{5}\right)-\left\{b_{5}, a_{5}, b_{2}, b_{3}\right\},
\end{aligned}
$$

$$
\begin{aligned}
& \mathrm{LR}_{8}=\operatorname{LR}\left(a_{3} a_{4}\right)=V\left(\mathrm{CDL}_{5}\right)-\left\{b_{1}, a_{1}, b_{3}, b_{4}\right\}, \\
& \operatorname{LR}_{9}=\operatorname{LR}\left(a_{4} a_{5}\right)=V\left(\mathrm{CDL}_{5}\right)-\left\{b_{2}, a_{2}, b_{4}, b_{5}\right\}, \\
& \operatorname{LR}_{10}=\operatorname{LR}\left(a_{5} a_{1}\right)=V\left(\mathrm{CDL}_{5}\right)-\left\{b_{1}, a_{3}, b_{3}, b_{5}\right\}, \\
& \operatorname{LR}_{11}=\operatorname{LR}\left(a_{1} b_{2}\right)=V\left(\mathrm{CDL}_{5}\right)-\left\{b_{4}, a_{4}, b_{1}, a_{2}\right\}, \\
& \operatorname{LR}_{12}=\operatorname{LR}\left(a_{2} b_{3}\right)=V\left(\mathrm{CDL}_{5}\right)-\left\{b_{5}, a_{5}, b_{2}, a_{3}\right\}, \\
& \operatorname{LR}_{12}=\operatorname{LR}\left(a_{3} b_{4}\right)=V\left(\mathrm{CDL}_{5}\right)-\left\{b_{1}, a_{4}, b_{3}, a_{4}\right\}, \\
& \operatorname{LR}_{11}=\operatorname{LR}\left(a_{1} b_{2}\right)=V\left(\mathrm{CDL}_{5}\right)-\left\{b_{4}, a_{4}, b_{1}, a_{2}\right\}, \\
& \operatorname{LR}_{15}=\operatorname{LR}\left(a_{5} b_{1}\right)=V\left(\mathrm{CDL}_{5}\right)-\left\{b_{3}, a_{3}, b_{5}, a_{6}\right\}, \\
& \operatorname{LR}_{16}=\operatorname{LR}\left(a_{1} b_{5}\right)=\left\{a_{1}, a_{2}, a_{4}, b_{2}, b_{4}, b_{4}\right\}, \\
& \operatorname{LR}_{17}=\operatorname{LR}\left(a_{2} b_{1}\right)=\left\{a_{2}, a_{3}, a_{5}, b_{1}, b_{3}, b_{5}\right\}, \\
& \operatorname{LR}_{18}=\operatorname{LR}\left(a_{3} b_{2}\right)=\left\{a_{2}, a_{3}, a_{4}, b_{1}, b_{2}, b_{4}\right\}, \\
& \operatorname{LR}_{19}=\operatorname{LR}\left(a_{4} b_{3}\right)=\left\{a_{2}, a_{4}, a_{5}, b_{2}, b_{3}, b_{5}\right\}, \\
& \operatorname{LR}_{20}=\operatorname{LR}\left(a_{5} b_{4}\right)=\left\{a_{1}, a_{3}, a_{5}, b_{1}, b_{3}, b_{4}\right\}, \\
& \operatorname{LR}_{21}=\operatorname{LR}\left(b_{1} a_{2}\right)=\left\{a_{2}, a_{3}, a_{5}, b_{1}, b_{3}, b_{5}\right\}, \\
& \operatorname{LR}_{22}=\operatorname{LR}\left(b_{2} a_{3}\right)=\left\{a_{1}, a_{3}, a_{4}, b_{1}, b_{2}, b_{4}\right\}, \\
& \operatorname{LR}_{23}=\operatorname{LR}\left(b_{3} a_{4}\right)=\left\{a_{2}, a_{4}, a_{5}, b_{2}, b_{3}, b_{5}\right\}, \\
& \operatorname{LR}_{24}=\operatorname{LR}\left(b_{4} a_{5}\right)=\left\{a_{1}, a_{3}, a_{5}, b_{1}, b_{3}, b_{4}\right\}, \\
& \operatorname{LR}_{25}=\operatorname{LR}\left(b_{5} a_{1}\right)=\left\{a_{1}, a_{2}, a_{4}, b_{2}, b_{4}, b_{5}\right\}, \\
& \operatorname{LR}_{26}=\operatorname{LR}\left(a_{1} b_{1}\right)=\left\{a_{1}, b_{1}\right\}, \\
& \operatorname{LR}_{27}=\operatorname{LR}\left(a_{2} b_{2}\right)=\left\{a_{2}, b_{2}\right\}, \\
& \operatorname{LR}_{28}=\operatorname{LR}\left(a_{3} b_{3}\right)=\left\{a_{3}, b_{3}\right\}, \\
& \left.\operatorname{LR}_{30}=\operatorname{LR}\left(a_{4} b_{4}\right)=\left\{a_{4}, b_{4}\right\}, b_{5}\right)=\left\{a_{5}, b_{5}\right\} .
\end{aligned}
$$

As, for $1 \leq i \leq 5, \quad\left|\operatorname{LRN}\left(e_{i}\right)\right|=2 \quad$ such that $\left|\operatorname{LR}\left(e_{i}\right)\right|<\left|\operatorname{LR}\left(e_{s}\right)\right|$, where $\quad 1 \leq s \leq 25$. Moreover, $\cup_{i=1}^{5} \operatorname{LR}\left(e_{i}\right)=V\left(\mathrm{CDL}_{5}\right)$; this implies $\left|\cup_{i=1}^{5} \operatorname{LR}\left(e_{i}\right)\right|=10$ and $\left|L R_{s} \cap \cup_{i=1}^{5} L R e_{i}\right| \geq\left|L R e_{i}\right|=2$. Define $\phi: V\left(\mathrm{CDL}_{5}\right)$ $\longrightarrow[0,1]$ such that $\phi(x)=(1 / 2)$ is the LRF with minimum cardinality for each $x \in V\left(\mathrm{CDL}_{5}\right)$. Consequently, by Proposition 1, $\operatorname{dim}_{l f}\left(\mathrm{CDL}_{5}\right)=\left(\left|V\left(\mathrm{CDL}_{5}\right)\right| / 2\right)=5$.

Case 3: For $n \geq 6$ and $1 \leq i \leq n$ by Lemma $1,\left|\operatorname{LR}\left(e_{i}\right)\right|=$ $2=\alpha$ and $\left|R \cap \cup_{i=1}^{n} \operatorname{LR}\left(e_{i}\right)\right| \geq 2$, where $\mathrm{R}$ are all other LRNs. Define $\phi: V\left(\mathrm{CDL}_{n}\right) \longrightarrow[0,1]$ such that $\phi$ is the LRF with minimum cardinality and $|\phi|<\left|\phi^{\prime}\right|$, as $\phi(v)=(1 / 2) \forall v \in V\left(\mathrm{CDL}_{n}\right) . \quad V\left(\mathrm{CDL}_{n}\right)=\cup\{\operatorname{LR}(e)$ : |LR $(e)=2\}$. Consequently, by Proposition 1, $\operatorname{dim}_{l f}\left(\mathrm{CDL}_{n}\right)=\left(\left|V\left(\mathrm{CDL}_{n}\right)\right| / 2\right)=(2 n / 2)=n$.

Theorem 4. Let $A_{n}$ with $n \geq 3$ be an antiprism network, where $n \cong 1(\bmod 2)$ and $\left|V\left(A_{n}\right)\right|=2 n$. Then, $\operatorname{dim}_{l f}\left(A_{n}\right)$,

$$
\frac{n}{n-1} \leq \operatorname{dim}_{l f}\left(A_{n}\right) \leq \frac{2 n}{n+1} \text {. }
$$

Proof. Case 1: For $n=3$, LRNs are as follows:

$$
\begin{aligned}
& \operatorname{LR}_{1}=\operatorname{LR}\left(a_{1} a_{2}\right)=\left\{a_{1}, a_{2}, b_{1}, b_{3}\right\}, \\
& \operatorname{LR}_{2}=\operatorname{LR}\left(a_{2} a_{3}\right)=\left\{a_{2}, a_{3}, b_{1}, b_{2}\right\}, \\
& \operatorname{LR}_{3}=\operatorname{LR}\left(a_{3} a_{1}\right)=\left\{a_{3}, a_{1}, b_{2}, b_{3}\right\},
\end{aligned}
$$




$$
\begin{aligned}
& \mathrm{LR}_{4}=\operatorname{LR}\left(a_{1} b_{1}\right)=\left\{a_{1}, a_{2}, b_{1}, b_{3}\right\}, \\
& \mathrm{LR}_{5}=\operatorname{LR}\left(a_{2} b_{2}\right)=\left\{a_{2}, a_{3}, b_{1}, b_{2}\right\}, \\
& \mathrm{LR}_{6}=\operatorname{LR}\left(a_{3} b_{3}\right)=\left\{a_{1}, a_{3}, b_{2}, b_{3}\right\}, \\
& \mathrm{LR}_{7}=\operatorname{LR}\left(a_{1} b_{2}\right)=\left\{a_{1}, a_{3}, b_{1}, b_{2}\right\}, \\
& \mathrm{LR}_{8}=\operatorname{LR}\left(a_{2} b_{3}\right)=\left\{a_{1}, a_{2}, b_{1}, b_{3}\right\}, \\
& \mathrm{LR}_{9}=\operatorname{LR}\left(a_{3} b_{1}\right)=\left\{a_{2}, a_{3}, b_{1}, b_{2}\right\} .
\end{aligned}
$$

As, for $1 \leq i \leq 9,\left|\operatorname{LR}\left(e_{i}\right)\right|=4$. Moreover, $\cup_{i=1}^{9} \operatorname{LR}\left(e_{i}\right)=$ $V\left(A_{3}\right)=8$ such that $\left|\cup_{i=1}^{9} \mathrm{LR}\left(e_{i}\right)\right|=6$ and $\mid \mathrm{LRN}_{s} \cap \cup_{i=1}^{9}$ $\operatorname{LR}\left(e_{i}\right)|>| \operatorname{LR}\left(e_{i}\right) \mid=4$. Define $\phi: V(A)_{3} \longrightarrow[0,1]$ as $\phi(x)=$ $(1 / 4)$ is the LRF with minimum cardinality. Consequently, by Corollary $1, \operatorname{dim}_{l f}\left(A_{3}\right)=\left(\left|V\left(A_{3}\right)\right| / 4\right)=(3 / 2)$.

Case 2: For $n=5$, LRNs are as follows:

$$
\begin{aligned}
& \mathrm{LR}_{1}=\operatorname{LR}\left(b_{1} b_{2}\right)=V\left(A_{5}\right)-\left\{a_{1}, b_{4}\right\}, \\
& \operatorname{LR}_{2}=\operatorname{LR}\left(b_{2} b_{3}\right)=V\left(A_{5}\right)-\left\{a_{3}, b_{5}\right\}, \\
& \operatorname{LR}_{3}=\operatorname{LR}\left(b_{3} b_{4}\right)=V\left(A_{5}\right)-\left\{a_{3}, b_{1}\right\}, \\
& \operatorname{LR}_{4}=\operatorname{LR}\left(b_{4} b_{5}\right)=V\left(A_{5}\right)-\left\{a_{4}, b_{2}\right\}, \\
& \operatorname{LR}_{5}=\operatorname{LR}\left(b_{5} b_{1}\right)=V\left(A_{5}\right)-\left\{a_{5}, b_{3}\right\}, \\
& \operatorname{LR}_{6}=\operatorname{LR}\left(a_{1} a_{2}\right)=V\left(A_{5}\right)-\left\{a_{4}, b_{2}\right\}, \\
& \operatorname{LR}_{7}=\operatorname{LR}\left(a_{2} a_{3}\right)=V\left(A_{5}\right)-\left\{a_{5}, b_{3}\right\}, \\
& \operatorname{LR}_{8}=\operatorname{LR}\left(a_{3} a_{4}\right)=V\left(A_{5}\right)-\left\{a_{1}, b_{4}\right\}, \\
& \operatorname{LR}_{9}=\operatorname{LR}\left(a_{4} a_{5}\right)=V\left(A_{5}\right)-\left\{a_{2}, b_{5}\right\}, \\
& \operatorname{LR}_{10}=\operatorname{LR}\left(a_{5} a_{1}\right)=V\left(A_{5}\right)-\left\{a_{3}, b_{1}\right\}, \\
& \operatorname{LR}_{11}=\operatorname{LR}\left(b_{1} a_{1}\right)=\left\{b_{1}, b_{4}, b_{5}, a_{1}, a_{2}, a_{3}\right\}, \\
& \operatorname{LR}_{12}=\operatorname{LR}\left(b_{2} a_{2}\right)=\left\{b_{2}, b_{5}, b_{1}, a_{2}, a_{3}, a_{4}\right\}, \\
& \operatorname{LR}_{13}=\operatorname{LR}\left(b_{3} a_{3}\right)=\left\{b_{3}, b_{1}, b_{2}, a_{3}, a_{4}, a_{5}\right\}, \\
& \operatorname{LR}_{14}=\operatorname{LR}\left(b_{4} a_{4}\right)=\left\{b_{4}, b_{2}, b_{1}, a_{4}, a_{5}, a_{1}\right\}, \\
& \operatorname{LR}_{15}=\operatorname{LR}\left(b_{5} a_{5}\right)=\left\{b_{5}, b_{2}, b_{3}, a_{5}, a_{1}, a_{2}\right\}, \\
& \operatorname{LR}_{16}=\operatorname{LR}\left(a_{1} b_{2}\right)=\left\{a_{1}, a_{4}, a_{5}, b_{2}, b_{3}, b_{4}\right\}, \\
& \operatorname{LR}_{17}=\operatorname{LR}\left(a_{2} b_{3}\right)=\left\{a_{2}, a_{5}, a_{1}, b_{3}, b_{4}, b_{5}\right\}, \\
& \operatorname{LR}_{18}=\operatorname{LR}\left(a_{3} b_{4}\right)=\left\{a_{3}, a_{1}, a_{2}, b_{4}, b_{5}, b_{1}\right\}, \\
& \operatorname{LR}_{19}=\operatorname{LR}\left(a_{4} b_{5}\right)=\left\{a_{4}, a_{2}, a_{1}, b_{5}, b_{1}, b_{2}\right\}, \\
& \operatorname{LR}_{20}=\operatorname{LR}\left(a_{5} b_{1}\right)=\left\{a_{5}, a_{2}, a_{3}, b_{1}, b_{2}, b_{3}\right\} .
\end{aligned}
$$

As, for $1 \leq i \leq 10, \quad\left|\operatorname{LR}\left(e_{i}\right)\right|=6 \quad$ such that $\left|\operatorname{LR}\left(e_{i}\right)\right|<\left|\operatorname{LR}\left(e_{s}\right)\right|$ and $1 \leq s \leq 10 . \cup_{i=1}^{10} \operatorname{LR}\left(e_{i}\right)=V\left(A_{5}\right)$; this implies $\quad\left|\cup_{i=1}^{10} \operatorname{LR}\left(e_{i}\right)\right|=10 \quad$ and $\quad \mid L R N \cap \cup_{i=1}^{10}$ $\mathrm{LR} e_{i}|>| \mathrm{LR} e_{i} \mid=6$. Define $\left(\phi: V(A)_{5}\right) \longrightarrow[0,1]$ as $\phi(x)=$ $(1 / 6)$ is the LRF with minimum cardinality for each $x \in V\left(A_{5}\right)$. Consequently, by Theorem 1, $\operatorname{dim}_{l f}\left(A_{5}\right) \leq\left(\left|V\left(A_{5}\right)\right| / 6\right) \leq(5 / 3)$.

As, for $1 \leq i \leq 10,\left|\operatorname{LR}\left(e_{i}\right)\right|=8,\left|\operatorname{LR}\left(e_{i}\right)\right|>\left|\operatorname{LR}\left(e_{s}\right)\right|$, and $1 \leq s \leq 10$. Moreover, $\quad \cup_{i=1}^{10} \operatorname{LR}\left(e_{i}\right)=V\left(A_{5}\right)$. Define $\left(\phi^{\prime}: V(A)_{5}\right) \longrightarrow[0,1]$ as $\phi^{\prime}(x)=(1 / 8)$ is the LRF with minimum cardinality for each $x \in V\left(A_{5}\right)$. Therefore, by Theorem 2, $\operatorname{dim}_{l f}\left(A_{5}\right) \geq\left(\left|V\left(A_{5}\right)\right| / 8\right) \geq(5 / 4)$. Consequently,

$$
\frac{5}{4} \leq \operatorname{dim}_{l f}\left(A_{5}\right) \leq \frac{5}{3} \text {. }
$$

Case 3: For $n \geq 6$ and $1 \leq i \leq n$ by Lemma $4,\left|\operatorname{LR}\left(e_{i}\right)\right|=$ $n+1=\alpha$ and $\left|R \cap \cup_{i=1}^{n} \operatorname{LR}\left(e_{i}\right)\right| \geq n+1$, where $\mathrm{R}$ are all other LRNs. There exists an ULRF $\phi: V\left(A_{n}\right) \longrightarrow[0,1]$, $|\phi|<\left|\phi^{\prime}\right|$ which is defined as $\phi(v)=(1 / \alpha) \forall v \in V\left(A_{n}\right)$, as $V\left(A_{n}\right)=\cup\{\operatorname{LR}(e): \mid \operatorname{LR}(e)=\alpha\}$. Consequently, by Theorem $1, \operatorname{dim}_{l f}\left(A_{n}\right) \leq\left(\left|V\left(A_{n}\right)\right| / n+1\right) \leq(2 n / n+1)$.

Case 4: For $\left|\operatorname{LR}\left(b_{i} b_{(i+1)}\right)\right|=2 n-2 \geq\left|\operatorname{LR}\left(e_{i}\right)\right|$, moreover, $\cup_{i=1}^{2 n} \operatorname{LR}\left(e_{i}\right)=V\left(A_{n}\right)$. There exists a LLRF such that $\quad \phi^{\prime}: V(A)_{n} \longrightarrow[0,1]$ is defined as $\phi^{\prime}(x)=(1 / 2 n-2)$ for each $x \in V\left(A_{n}\right)$. Therefore, by Theorem 2, we have $\operatorname{dim}_{l f}\left(A_{n}\right) \geq(n / n-1)$. Consequently,

$$
\frac{n}{n-1} \leq \operatorname{dim}_{l f}\left(A_{n}\right) \leq \frac{2 n}{n+1} .
$$

Theorem 5. Let $A_{n}$ with $n \geq 4$ be an antiprism network, where $n \cong 0(\bmod 2)$ and $\left|V\left(A_{n}\right)\right|=2 n$. Then,

$$
\frac{n}{n-1} \leq \operatorname{dim}_{l f}\left(A_{4}\right) \leq 2 \text {. }
$$

Proof. Case 1: For $n=4$, LRNs are as follows:

$$
\begin{aligned}
& \mathrm{LR}_{1}=\mathrm{LR}\left(b_{1} b_{2}\right)=V\left(A_{4}\right)-\left\{a_{1}, a_{3}\right\}, \\
& \mathrm{LR}_{2}=\mathrm{LR}\left(b_{2} b_{3}\right)=V\left(A_{4}\right)-\left\{a_{2}, a_{4}\right\}, \\
& \mathrm{LR}_{3}=\operatorname{LR}\left(b_{3} b_{4}\right)=V\left(A_{4}\right)-\left\{a_{1}, a_{3}\right\}, \\
& \mathrm{LR}_{4}=\operatorname{LR}\left(b_{4} b_{1}\right)=V\left(A_{4}\right)-\left\{a_{2}, a_{4}\right\}, \\
& \mathrm{LR}_{5}=\operatorname{LR}\left(a_{1} a_{2}\right)=V\left(A_{4}\right)-\left\{b_{2}, b_{4}\right\}, \\
& \mathrm{LR}_{6}=\operatorname{LR}\left(a_{2} a_{3}\right)=V\left(A_{4}\right)-\left\{b_{3}, b_{1}\right\}, \\
& \mathrm{LR}_{7}=\operatorname{LR}\left(a_{3} a_{4}\right)=V\left(A_{4}\right)-\left\{b_{2}, b_{4}\right\}, \\
& \mathrm{LR}_{8}=\operatorname{LR}\left(a_{4} a_{1}\right)=V\left(A_{4}\right)-\left\{b_{1}, b_{3}\right\}, \\
& \mathrm{LR}_{9}=\operatorname{LR}\left(b_{1} a_{1}\right)=\left\{b_{1}, b_{4}, a_{1}, a_{2}\right\}, \\
& \mathrm{LR}_{10}=\operatorname{LR}\left(b_{2} a_{2}\right)=\left\{b_{1}, b_{2}, a_{2}, a_{3}\right\}, \\
& \mathrm{LR}_{11}=\operatorname{LR}\left(b_{3} a_{3}\right)=\left\{b_{2}, b_{3}, a_{3}, a_{4}\right\}, \\
& \operatorname{LR}_{12}=\operatorname{LR}\left(b_{4} a_{4}\right)=\left\{b_{3}, b_{4}, a_{1}, a_{4}\right\}, \\
& \operatorname{LR}_{13}=\operatorname{LR}\left(b_{2} a_{1}\right)=\left\{b_{2}, b_{3}, a_{1}, a_{4}\right\}, \\
& \operatorname{LR}_{14}=\operatorname{LR}\left(b_{3} a_{2}\right)=\left\{b_{3}, b_{4}, a_{1}, a_{2}\right\}, \\
& \operatorname{LR}_{15}=\operatorname{LR}\left(b_{4} a_{3}\right)=\left\{b_{1}, b_{4}, a_{2}, a_{3}\right\}, \\
& \operatorname{LR}_{16}=\operatorname{LR}\left(b_{1} a_{4}\right)=\left\{b_{1}, b_{2}, a_{3}, a_{4}\right\} .
\end{aligned}
$$

As, for $\quad 1 \leq i \leq 8, \quad\left|L R\left(e_{i}\right)\right|=4 \quad$ such that $\left|\operatorname{LR}\left(e_{i}\right)\right|<\left|\operatorname{LR}\left(e_{s}\right)\right|$, where $1 \leq s \leq 8$. Moreover, $\cup_{i=1}^{8} \operatorname{LR}\left(e_{i}\right)=$ $V\left(A_{4}\right)$ and $\left|\mathrm{LR}_{s} \cap \cup_{i=1}^{8} \operatorname{LR}\left(e_{i}\right)\right|>\left|\mathrm{LR}_{i}\right|=4$. There exits an ULRF $\phi: V(A)_{4} \longrightarrow[0,1]$ defined as $\phi(x)=(1 / 4)$ which is the LRF with minimum cardinality $\forall x \in V\left(A_{4}\right)$. Consequently, by Theorem $1, \operatorname{dim}_{l f}\left(A_{4}\right) \leq\left(\left|V\left(A_{4}\right)\right| / 4\right) \leq 2$.

As, for $1 \leq i \leq 8, \quad\left|\operatorname{LR}\left(e_{i}\right)\right|=6 \quad$ such that $\left|\operatorname{LR}\left(e_{i}\right)\right|>\left|\operatorname{LR}\left(e_{s}\right)\right|, \quad$ where $1 \leq s \leq 8 . \quad$ Moreover, $\quad \cup_{i=1}^{8}$ $\operatorname{LR}\left(e_{i}\right)=V\left(A_{4}\right)$. There exists a LLRF $s \phi^{\prime}: V\left(A_{4}\right) \longrightarrow[0,1]$ which is defined as $\phi^{\prime}(x)=(1 / 6)$ for each $x \in V\left(A_{4}\right)$. Therefore, by Theorem 1, we have $\operatorname{dim}_{l f}$ $\left(A_{4}\right) \geq\left(V\left(A_{4}\right) / 6\right)(4 / 3)$. Consequently,

$$
\frac{4}{3} \leq \operatorname{dim}_{l f}\left(A_{4}\right) \leq 2 .
$$


TABLE 1: LFMD of networks.

\begin{tabular}{lcr}
\hline Networks & LFMDs & Comment \\
\hline Antiprism network $\left[A_{n}\right.$ for $\left.n \cong 0(\bmod 2)\right]$ & $(n / n-1) \leq \operatorname{dim}_{l f}\left(A_{n}\right) \leq 2$. & Bounded \\
Antiprism network $\mathrm{A}_{n}$, where $n \cong 1(\bmod 2)$ & $(n / n-1) \leq \operatorname{dim}_{l f}\left(A_{n}\right) \leq(2 n / n+1)$. & Bounded \\
Sun flower network $\mathrm{SF}_{n}$. & $(n / n-1) \leq \operatorname{dim}_{l f}\left(\mathrm{SF}_{n}\right) \leq(2 n / n+1)$. & Bounded
\end{tabular}

Case 2: For $n \geq 5,1 \leq i \leq n$, by Lemma 3, $\left|\operatorname{LR}\left(e_{i}\right)\right|=n=$ $\alpha$ and $\left|R \cap \cup_{i=1}^{n} \operatorname{LR}\left(e_{i}\right)\right| \geq n$, where $\mathrm{R}$ are all other LRNs.

There exists an ULRF $\phi: V\left(A_{n}\right) \longrightarrow[0,1]$ which is defined as $\phi(v)=(1 / \alpha) \forall v \in V\left(A_{n}\right)$, as $\mathrm{V}(\mathrm{G})$ $=\bigcup\{\operatorname{LR}(e): \mid \operatorname{LR}(e)=\alpha\}$. Consequently, by Theorem 1 , $\operatorname{dim}_{l f}\left(A_{n}\right) \leq\left(\left|V\left(A_{n}\right)\right| / n\right) \leq(2 n / n)=2$.

Case 3: As, $\left|\operatorname{LR}\left(a_{i} a_{(i+1)}\right)\right|=2 n-2$, which is greater or equal to the cardinalities of all other LRNs, moreover, $\cup_{i=1}^{2 n} \operatorname{LR}\left(e_{i}\right)=V\left(A_{n}\right)$. There exists a LLRF $\left(\phi^{\prime}: V(A)_{n}\right) \longrightarrow[0,1]$ which is defined as $\phi^{\prime}(x)=(1 / 2 n-2) \forall v \in V\left(A_{n}\right)$. Therefore, by Theorem $2, \operatorname{dim}_{l f}\left(A_{n}\right) \geq(n / n-1)$. Consequently,

$$
\frac{n}{n-1} \leq \operatorname{dim}_{l f}\left(A_{n}\right) \leq 2 \text {. }
$$

Theorem 6. Let $S F_{n}$ with $n \geq 3$ be a sun flower network and $\left|V\left(S F_{n}\right)\right|=2 n$. Then,

$$
\frac{n}{n-1} \leq \operatorname{dim}_{l f}\left(\mathrm{SF}_{n}\right) \leq \frac{2 n}{n+1} .
$$

Proof. Case 1: For $n=3$, LRNs are as follows:

$$
\begin{aligned}
& \mathrm{LR}_{1}=\operatorname{LR}\left(a_{1} a_{2}\right)=\left\{a_{1}, a_{2}, b_{2}, b_{3}\right\}, \\
& \operatorname{LR}_{2}=\operatorname{LR}\left(a_{2} a_{3}\right)=\left\{a_{2}, a_{3}, b_{3}, b_{1}\right\}, \\
& \mathrm{LR}_{3}=\operatorname{LR}\left(a_{3} a_{1}\right)=\left\{a_{3}, a_{1}, b_{1}, b_{2}\right\}, \\
& \mathrm{LR}_{4}=\operatorname{LR}\left(a_{1} b_{1}\right)=\left\{a_{1}, a_{3}, b_{1}, b_{3}\right\}, \\
& \operatorname{LR}_{5}=\operatorname{LR}\left(a_{2} b_{2}\right)=\left\{a_{1}, a_{2}, b_{1}, b_{2}\right\}, \\
& \operatorname{LR}_{6}=\operatorname{LR}\left(a_{3} b_{3}\right)=\left\{a_{2}, a_{3}, b_{2}, b_{3}\right\}, \\
& \operatorname{LR}_{7}=\operatorname{LR}\left(a_{2} b_{1}\right)=\left\{b_{1}, b_{2}, a_{2}, a_{3}\right\}, \\
& \operatorname{LR}_{8}=\operatorname{LR}\left(a_{3} b_{2}\right)=\left\{a_{1}, a_{3}, b_{2}, b_{3}\right\}, \\
& \operatorname{LR}_{9}=\operatorname{LR}\left(a_{1} b_{3}\right)=\left\{a_{1}, a_{2}, b_{1}, b_{3}\right\} .
\end{aligned}
$$

As, for $\quad 1 \leq i \leq 9, \quad\left|\operatorname{LR}\left(e_{i}\right)\right|=4 \quad$ and $\left|\operatorname{LRN} s \cap \cup_{i=1}^{9} \operatorname{LR}\left(e_{i}\right)\right|=\left|\operatorname{LR}\left(e_{i}\right)\right|=4$. There exists an ULRF $\phi: V\left(\mathrm{SF}_{3}\right) \longrightarrow[0,1]$ which is defined by $\phi(x)=(1 / 4)$, $\forall v \in V\left(\mathrm{SF}_{3}\right)$. Consequently, by Corollary 1 , $\operatorname{dim}_{l f}\left(\mathrm{SF}_{3}\right)=(3 / 2)$.

Case 2: For $n=4$, LRNs are as follows:

$$
\begin{aligned}
& \operatorname{LR}_{1}=\operatorname{LR}\left(a_{1} a_{2}\right)=\left\{a_{1}, a_{2}, a_{3}, a_{4}, b_{2}, b_{4}\right\}, \\
& \operatorname{LR}_{2}=\operatorname{LR}\left(a_{2} a_{3}\right)=\left\{a_{1}, a_{2}, a_{3}, a_{4}, b_{1}, b_{3}\right\}, \\
& \operatorname{LR}_{3}=\operatorname{LR}\left(a_{3} a_{4}\right)=\left\{a_{1}, a_{2}, a_{3}, a_{4}, b_{2}, b_{4}\right\}, \\
& \operatorname{LR}_{4}=\operatorname{LR}\left(a_{4} a_{1}\right)=\left\{a_{1}, a_{2}, a_{3}, a_{4}, b_{1}, b_{3}\right\}, \\
& \operatorname{LR}_{5}=\operatorname{LR}\left(a_{1} b_{1}\right)=\left\{a_{1}, a_{4}, b_{1}, b_{2}, b_{4}\right\}, \\
& \operatorname{LR}_{6}=\operatorname{LR}\left(a_{2} b_{2}\right)=\left\{a_{1}, a_{2}, b_{1}, b_{2}, b_{4}\right\}, \\
& \operatorname{LR}_{7}=\operatorname{LR}\left(a_{3} b_{3}\right)=\left\{a_{2}, a_{3}, b_{1}, b_{2}, b_{3}\right\},
\end{aligned}
$$

$$
\begin{aligned}
& \operatorname{LR}_{8}=\operatorname{LR}\left(a_{4} b_{4}\right)=\left\{a_{3}, a_{4}, b_{2}, b_{3}, b_{4}\right\}, \\
& \operatorname{LR}_{9}=\operatorname{LR}\left(a_{2} b_{1}\right)=\left\{a_{2}, a_{3}, b_{1}, b_{2}, b_{3}\right\}, \\
& \operatorname{LR}_{10}=\operatorname{LR}\left(a_{3} b_{2}\right)=\left\{a_{3}, a_{4}, b_{2}, b_{3}, b_{4}\right\}, \\
& \operatorname{LR}_{11}=\operatorname{LR}\left(a_{4} b_{3}\right)=\left\{a_{1}, a_{4}, b_{2}, b_{3}, b_{4}\right\}, \\
& \operatorname{LR}_{12}=\operatorname{LR}\left(a_{1} b_{4}\right)=\left\{a_{1}, a_{2}, b_{1}, b_{2}, b_{4}\right\} .
\end{aligned}
$$

As, for $1 \leq i \leq 8, \quad\left|\operatorname{LR}\left(e_{i}\right)\right|=5, \quad$ such that $\left|\operatorname{LR}\left(e_{i}\right)\right|<\left|\operatorname{LR}\left(e_{s}\right)\right|$, where $\quad 1 \leq s \leq 4$. Moreover, $\cup_{i=1}^{8} \mathrm{LR}\left(e_{i}\right)=V\left(\mathrm{SF}_{4}\right)$. There exists $\left(\phi: V(\mathrm{SF})_{4}\right) \longrightarrow[0,1]$ defined as $\phi(x)=(1 / 5)$ which is the LRF $\forall v \in V\left(\mathrm{SF}_{4}\right)$, as $V\left(\mathrm{SF}_{4}\right)=\cup\left\{\operatorname{LR}\left(e_{i}\right): \mid \operatorname{LR}(e)=5\right\}$. Consequently, by Theorem $1, \operatorname{dim}_{l f}\left(\mathrm{SF}_{4}\right) \leq(8 / 5)$.

As, for $1 \leq i \leq 4, \quad\left|\operatorname{LR}\left(e_{i}\right)\right|=6 \quad$ such that $\left|\operatorname{LR}\left(e_{i}\right)\right|>\left|\operatorname{LR}\left(e_{s}\right)\right|$, where $1 \leq s \leq 8$. There exists $\phi^{\prime}: V\left(\mathrm{SF}_{4}\right) \longrightarrow[0,1]$ defined as $\phi(v)=(1 / 6) \forall v \in V\left(\mathrm{SF}_{4}\right)$, which is the LLRF with maximum cardinality, where $\left|\phi^{\prime}\right|<\left|\phi^{\prime \prime}\right|$, as $V\left(\mathrm{SF}_{4}\right)=\bigcup\{\operatorname{LR}(e): \mid \operatorname{LR}(e)=6\}$. By Theorem $1, \operatorname{dim}_{l f}\left(\mathrm{SF}_{4}\right) \geq(4 / 3)$. Consequently,

$$
\frac{4}{3} \leq \operatorname{dim}_{l f}\left(\mathrm{SF}_{4}\right) \leq \frac{8}{5}
$$

Case 3: As $n \geq 5,1 \leq i \leq n,\left|\operatorname{LR}\left(e_{i}\right)\right|=n+1=\alpha$, and $\left|R \cap \cup_{i=1}^{n} \operatorname{LR}\left(e_{i}\right)\right| \geq n+1$, where $\mathrm{R}$ are all other LRNs. There exists an ULRF $\phi: V\left(\mathrm{SF}_{n}\right) \longrightarrow[0,1]$ defined as $\phi(v)=(1 / \alpha) \forall v \in V\left(\mathrm{SF}_{n}\right), \quad$ as $\quad \mathrm{V}(\mathrm{G})$ $=\cup\{\operatorname{LR}(e): \mid \operatorname{LR}(e)=\alpha\}$. Consequently, by Theorem 1 , $\operatorname{dim}_{l f}\left(\mathrm{SF}_{n}\right) \leq\left(\left|V\left(\mathrm{SF}_{n}\right)\right| / n+1\right) \leq(2 n / n+1)$.

Case 4: As $\left|\operatorname{LR}\left(a_{i} a_{i+1}\right)\right|=2 n-2$ which is greater than the cardinalities of all other LRNs of $\mathrm{SF}_{n}$, there exists a LLRF $\quad \phi^{\prime}: V\left(\mathrm{SF}_{n}\right) \longrightarrow[0,1]$ defined by $\phi^{\prime}(v)=(1 / 2 n-2), \quad \forall v \in V\left(S F_{n}\right), \quad$ as $\quad \mathrm{V}(\mathrm{G})$ $=\cup\{\operatorname{LR}(e): \mid \operatorname{LR}(e)=2 n\}$. By Theorem 2, $\operatorname{dim}_{l f}\left(\mathrm{SF}_{n}\right) \geq(n / n-1)$. Consequently,

$$
\frac{n}{n-1} \leq \operatorname{dim}_{l f}\left(\mathrm{SF}_{n}\right) \leq \frac{2 n}{n+1} .
$$

\section{Conclusion}

In this article, we studied the various aspects of the LFMDs for the different connected networks including the existence of the exact values, lower and upper bonds and bounded- and unbounded behaviours. Mainly, the lower bond of LFMD for the arbitrary connected networks is improved from unity. As the applications of the main result, LFMDs of the prism-related networks are as follows:

(i) The exact value LFMD of $C D L_{n}$ is $n$ and of $A_{3}$ is (3/2), where $n \geq 4$. 
(ii) The LFMDs of antiprism and sun flower networks are computed in bounds, see Table 1.

Moreover, we note that, for $n \longrightarrow \infty$, the computed bounds are convergent which show their boundedness. Now, we close our discussion by proposing the following open problem: open problem. Characterize the connected networks whose LFMDs is $(|V(G)| / a)$, where $a<|V(G)|$ is some integral value.

\section{Data Availability}

The data used to support the findings of this study are included within this article. However, the reader may contact the corresponding author for more details on the data.

\section{Conflicts of Interest}

The authors have no conflicts of interest.

\section{References}

[1] P. J. Slater, "Leaves of trees," Congressus Numerantium, vol. 14, no. 1, pp. 549-559, 1975.

[2] F. Harary and R. Melter, "On the metric dimension of a graph,” Ars Combinatoria, vol. 2, pp. 19-195, 1976.

[3] J. Currie and O. R. Oellermann, "The metric dimension and metric independence of a graph," Journal of Combinatorial Mathematics and Combinatorial Computing, vol. 39, pp. 157-167, 2001.

[4] Y. Shang, "Percolation in a hierarchical lattice," Zeitschrift Natur-Forschung, vol. 6, 2012.

[5] S. Khuller, B. Raghavachari, and A. Rosenfeld, Localization in Graphs, Technical Report UMIACS-TR-94-92, University of Maryland, College Park, MD, USA, 1994.

[6] G. Chartrand, L. Eroh, M. A. Johnson, and O. R. Oellermann, "Resolvability in graphs and the metric dimension of a graph," Discrete Applied Mathematics, vol. 105, no. 1-3, pp. 99-113, 2000.

[7] M. Fehr, S. Gosselin, and O. R. Oellermann, "The metric dimension of Cayley digraphs," Discrete Mathematics, vol. 306, no. 1, pp. 31-41, 2006.

[8] Y.-M. Chu, M. F. Nadeem, M. Azeem, and M. K. Siddiqui, "On sharp bounds on partition dimension of convex polytopes,” IEEE Access, vol. 8, pp. 224781-224790, 2020.

[9] J. B. Liu and M. F. Nadeem, "Bounds on the partition dimension of convex polytopes," Combinatorial Chemistry and High throughout Screening, inprint, 2020.

[10] J.-B. Liu, M. F. Nadeem, H. M. A. Siddiqui, and W. Nazir, "Computing metric dimension of Certain families of toeplitz graphs,” IEEE Access, vol. 7, pp. 126734-126741, 2019.

[11] Z. S. Mufti, M. F. Nadeem, A. Ahmad, and Z. Ahmad, "Computation of edge metric dimension of barycentric subdivision of cayley graphs," Italian Journal of Pure and Applied Mathematics, vol. 44, pp. 714-722, 2020.

[12] S. Arumugam and V. Mathew, "The fractional metric dimension of graphs," Discrete Mathematics, vol. 312, no. 9, pp. 1584-1590, 2012.

[13] M. Feng and K. Wang, "On the metric dimension and fractional metric dimension for hierarchical product of graphs," Applicable Analysis and Discrete Mathematics, vol. 7, no. 2, pp. 302-313, 2013.
[14] J. B. Liu, A. Kashif, T. Rashid, and M. Javaid, "Fractional metric dimension of generalized Jahangir graph," Mathematics, vol. 7, pp. 1-10, 2019.

[15] S. Aisyah, M. Utoyo, and L. Susilowati, "On the local fractional metric dimension of corona product networks, IOP Conference," Earth and Environmental Science Hungarica, vol. 243, pp. 1-4, 2019.

[16] J.-B. Liu, M. K. Aslam, and M. Javaid, "Local fractional metric dimensions of rotationally symmetric and planar networks," IEEE Access, vol. 8, pp. 82404-82420, 2020.

[17] M. Javaid, S. Safdar, M. U. Farooq, and M. K. Aslam, "Computing sharp bounds for local fractional metric dimensions of cycle related graphs," Computational Journal of Combinatorial Mathematics, vol. 1, pp. 29-73, 2020.

[18] M. Javaid, M. Raza, P. Kumam, and J.-B. Liu, "Sharp bounds of local fractional metric dimensions of connected networks," IEEE Access, vol. 8, pp. 172329-172342, 2020.

[19] S. Yani, E. Iwan, and S. Budi, On Some New Edge Odd Gracful Graphs, University of Gadijah Moda, Yogyakarta, Indonasia, 2019. 